\title{
Effects of the Ionosphere on VLF Navigational Aids ${ }^{1}$
}

\author{
W. T. Blackband \\ Contribution from Radio Department, Royal Aircraft Establishment, Farnborough, Hants, England
}

(Received November 8, 1960; revised June 30, 1961)

\begin{abstract}
Long range navigational aids could be devised using VLF transmissions. The propagation of such waves is controlled by the lowest level of the ionosphere. The small changes in phase velocity which accompany the diurnal ionospheric changes have been studied using ground monitor stations. Preliminary measurements made in an aircraft show internal consistency in fixing of about 1 nautical mile at ranges of 5,000 to 6,000 miles.
\end{abstract}

\section{Introduction}

The very low frequencies are those between 3 and $30 \mathrm{kc} / \mathrm{s}$. 'This paper will not be concerned with any specific navigational aids but will discuss those factors of VLF propagation which will dominate the performance of any aids operating in this frequency band. It must be stressed that this description of the propagation should not be extrapolated into adjacent bands, and in particular would not apply without some considerable modification to the frequency band 70 to $120 \mathrm{kc} / \mathrm{s}$.

In the VLF waveband the absorption at the ionosphere on reflection at large angles of incidence is small at all times of day and consequently propagation is possible even in daylight at $16 \mathrm{kc} / \mathrm{s}$ under circumstances in which there would be strong ionospheric absorption a few decades higher in frequency. For this reason propagation losses will not be discussed in detail and attention will be concentrated on the phase velocity of propagation of the signals because it is this velocity which determines the measured range from the transmitter in any phase comparison navigational aid. This velocity determines the wavelength which is the unit in which all measurements of distance are made. In general it is easier to observe changes in this velocity rather than determine its absolute value.

In discussion of VLF propagation we are concerned only with the lowest part of the ionosphere - the $D$ layer. The apparent height at nighttime is about 92 $\mathrm{km}$ while that at daytime is generally in the region of 72 to $80 \mathrm{~km}$. For waves polarized with their electric vector normal to the ionosphere (vertically polarized), and at large angles of incidence, the signal is reflected without change in polarization, and the ionosphere acts as a sharply bounded conducting layer. Most VLF transmitting aerials radiate vertically polarized signals, and consequently there is little restriction in practice through confining attention in this paper to vertically polarized rays.

1 This is a revised version of a paper presented at the Avionics Panel Meeting of the Advisory Group for Aeronautical Research and Development (AGARD) NATO, Istanbul, Turkey, October 1960.

\section{Mechanisms of Propagation}

The VLF band is of interest for navigational aids because of the long distance propagation which could permit the development of an aid of worldwide usefulness. The long-range propagation beyond the line of sight is achieved through repeated reflection of the transmitted wave between ionosphere and earth. This is illustrated in figure 1 which shows how, although the transmitter $T$ is not directly visible from the receiver $R$, signals can be received having made one or two hops by way of the ionosphere which at these frequencies acts as a reflecting layer. Signals could reach $R$ by a path involving many reflectionssuch as that shown by a dotted line, however because the reflection coefficient is small for small angles of incidence such signals would be rapidly attenuated, and the number of ray paths capable of transmitting an appreciable signal level to $R$ is limited. It is usual to consider the propagation between earth and ionosphere in terms of the several ray paths for propagation up to 1,200 or $1,500 \mathrm{~km}$. By taking into account the reflection coefficients of earth and ionosphere, it is possible by ray theory to explain [Wait and

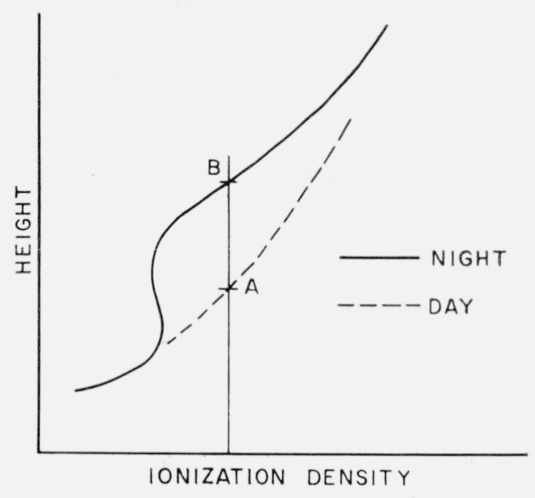

FIgURE 1. Ionization contour for lower ionosphere ( $D$ region). 
Murphy, 1957] the main features of this mediumrange propagation including the maxima and minima in signal corresponding to the Hollingsworth pattern of higher frequencies. These maxima and minima appear in figure 2 which shows a plot of field strength of GBR Rugby on $16 \mathrm{kc} / \mathrm{s}$ against great circle distance from the transmitter. This measurement was made in an aircraft flying at 40,000 ft from Farnborough, to Malta in daylight. This shows a minimum at about 230 nautical miles which arises through interference between the ground ray and first hop skywave of figure 1, and a second minimum at 740 nautical miles arising through interference between first and second hop skywaves.

At distances greater than about $1,200 \mathrm{~km}$ it is usual to describe propagation in terms of waveguide modes [Wait, 1957a; Budden, 1957]. The earth and ionosphere are then considered as the conducting walls of a waveguide. This waveguide differs from those in general use because these conducting walls are relatively far apart (about $2 \frac{1}{2} \lambda$ at $10 \mathrm{kc} / \mathrm{s}$ and $8 \lambda$ at $30 \mathrm{kc} / \mathrm{s}$ ) and consequently several modes can be transmitted. If both earth and ionosphere were perfect conductors the distributions would be true sine waves. Thus we have the first mode which has a half sine wave distribution of voltage vector between ionosphere and earth, and the higher order modes which have distributions following several half sine waves. However, there are resistive losses in both earth and ionosphere and consequently the waves penetrate the waveguide walls. This results in a distortion from the true sine wave and some bending of the wavefront from the vertical. The losses in the ground and ionosphere are most marked for the higher order modes and consequently these modes are reduced to low amplitudes before they have traveled far. As a result of this the first mode usually dominates at distances of $2,000 \mathrm{~km}$ or more although for some ionospheric conditions the zero order mode may be the most important at the $3 \mathrm{kc} / \mathrm{s}$ end of the frequency band.

\section{Attenuation of Signals}

This is a facet of the subject of great complexity. Towards the lower end of the VLF band there is marked absorption while above $10 \mathrm{kc} / \mathrm{s}$ the attenuation is of the order of $2 \mathrm{db} / 1,000 \mathrm{~km}$. In general the

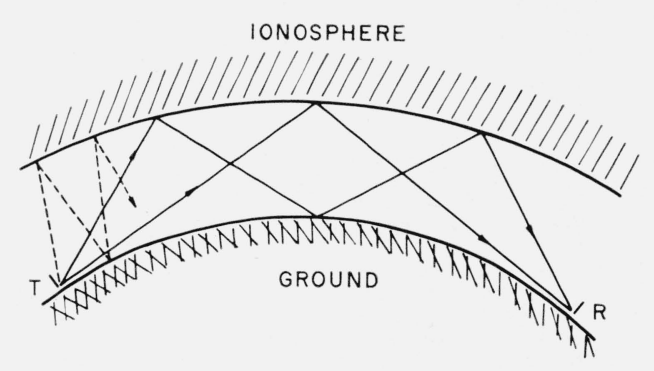

Figure 2. Ionospheric reflection of VLF waves. losses for transmission paths over sea are less than those for a corresponding overland path by a factor of as much as 2:1. A detailed theoretical study [Wait, 1957b] has been presented which expresses the frequency variation of attenuation for each mode in terms of the height of the ionosphere, and the conductivities of ground and ionosphere. Inasmuch as it is difficult to determine the properties of the lowest layers of the ionosphere except by VLF studies, it is not easy to confirm these theoretical predictions in detail. However, there is no doubt that they are a useful guide in the interpretation of the experimental phenomena. Figure 3 shows the measured variation of signal with distance for an over sea path from San Diego-Hawaii compared with that which would be predicted theoretically [Wait, 1957a] on the basis of chosen parameters for the ionosphere. The similarity between the forms of the two curves is pleasing, and the general agreement is as good as could be expected bearing in mind the somewhat arbitrary assumptions made about the ionosphere.

\section{Velocity}

In any navigational system at present contemplated the observations will take the form of phase comparisons made either between a number of received signals, or between received signals and a high stability oscillator carried by the aircraft. For this reason we are interested in the phase velocity of the total wave. At distances from the transmitter for which the waveguide mode theory is applicable it is to be expected that the phase velocity would be somewhat greater than unity because of the finite spacing of ground and ionosphere, on the other hand, the effect of losses in the waveguide walls would be to slow down the wave. Some measured values have been published.

In the region near to the transmitter the effective phase velocity at any range can be deduced from the variation with radial distance of the resultant phase of the ground and sky rays, allowance being made for their differing ray paths and the changes of phase and amplitude on reflection.

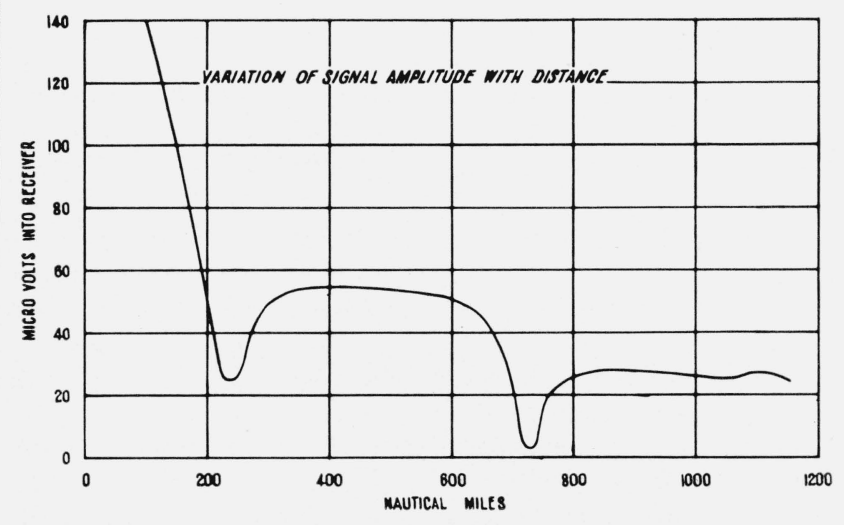

Figure 3. Variation of signal amplitude with distance. 


\section{Diurnal Changes in Phase Over a Given Path}

If the phase of a signal received at a distant point is compared with that of a standard oscillator, then changes in velocity of propagation will become apparent as variations in relative phase. It has been found that there are diurnal variations in the phase of the received signal associated with changes from daylight to darkness. These are well illustrated in figure 4 in which J. A. Pierce [1957] depicts his "phase trapezium." The far left of the figure refers to times at which the whole $5,200-\mathrm{km}$ path between the transmitter at Rugby and the receiver at Cambridge, Mass., is in darkness. At a short time before sunrise at Rugby a steady phase change commences, ending as the sun rises at Cambridge. During the day the phase remains constant until sunset at Rugby when the reverse process begins until the steady night value is reached at Cambridge sunset. The phase change is in a sense corresponding to a daytime increase in phase velocity. It is explained as the result of a reduction of the apparent height of the ionosphere during the day. This would be accounted for on the ray theory by saying that with the lower ionosphere the sky rays had a shorter path, or on the basis of waveguide modes, that the phase velocity had been increased by bringing the waveguide walls closer together.

It may be noted that the sharp onset of these phase changes occurs not at ground sunrise but a little earlier, at the time at which the sun viewed from a height of about $90 \mathrm{~km}$ above the receiver would be seen to rise above the ozonosphere.

The phase change of figure 4 has been found to be predictable from day to day within a few microseconds - an uncertainty in prediction which would introduce a positional error of about 1 mile in a navigational aid on a transatlantic flight. A series of measurements of the diurnal phase changes of the signals received from GBR have been made at Malta. These have shown variations which were basically repeatable from day to day but were more complex than those reported by Pierce. The changes for one day is shown in figure 5 from which it will be noted that instead of the neat trapezium there is a slight reverse drift before dawn followed by an overswing of phase before the steady day time value is reached. It has been suggested that these phase swings arise through the beating of signals reflected from two layers or that they represent the variations in phase of the sum of several modes, each mode being differently affected by the changing ionosphere. It would be expected that such overswing would be most apparent on a path which lay roughly parallel to the dawn or dusk line, because for this dawn or dusk would occur almost simultaneously along the whole path, all parts then being in the same intermediate state between day and night. For other paths where at any time parts are in different intermediate states, the overswings are averaged out. Even if the path follows the dawn of dusk line the effect of overswings would not be expected to appear

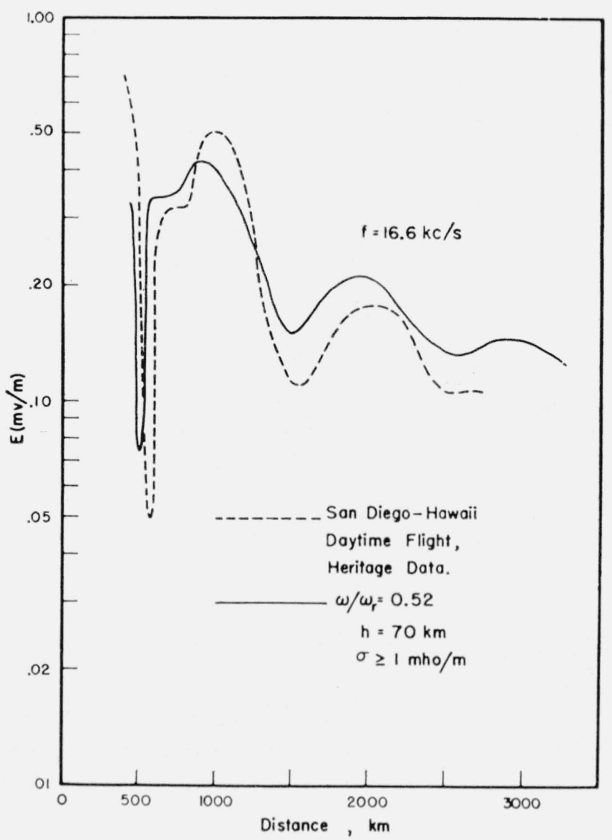

Figure 4. Comparison of measured and computed field strengths (after Wait, Proc. IRE, June 195\%).

An experimental field-strength-versus-distance curve for $16.6 \mathrm{kc} / \mathrm{s}$ over sea and the corresponding calculated curve assuming $\omega / \omega_{r}=0.52$ or $\omega_{r}=2.0 \times 10^{5}, h=70 \mathrm{~km}$ and $\sigma \geq 1$ mho/meter.

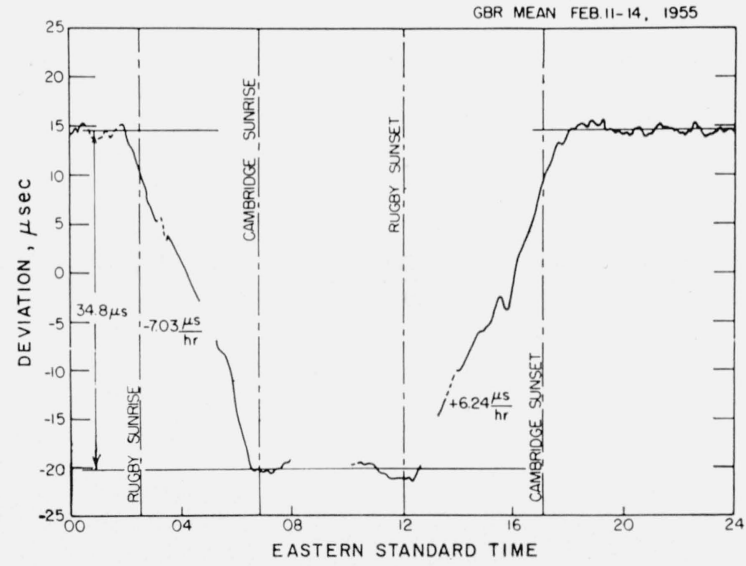

Figure 5. The diurnal variation of the time of arrival of the GBR signal at Cambridge, Mass. (after Pierce, Proc. IRE, June 195\%).

at distances so great that only a single mode is received at an appreciable amplitude. At present it is not possible to check these theories because sufficient data are not available.

The magnitude of the diurnal phase change varies with distance from the transmitter. At distant receivers the ground ray will be negligible, and in order to compare signals near to the transmitter with those received at a distance it will be most convenient to neglect the ground ray and consider only the resultant sky ray. 
At a point near to the transmitter the sky ray will be reflected at near normal incidence, and so when the reflecting layer changes by $18 \mathrm{~km}$ in apparent height from day to night the sky-ray path will change by $36 \mathrm{~km}$ - or a time of say $120 \mu$ sec. As the point of reception is moved further away from the transmitter the change in sky-ray path length will decrease rapidly at first and then more slowly as glancing incidence is approached. For greater distances the waveguide mode theory would predict a linear increase in phase change with distance. The initial rapid fall and linear rise would combine to give a variation of phase change with distance of the form of figure 6. Various measured values of phase change have been plotted and show a reasonable agreement with the expected shape of the curve. If this curve can be established by further measurement at other ranges up to say $10,000 \mathrm{~km}$, then the diurnal variations of phase for a VLF navagational aid can be predicted with accuracy.

Such programs of monitoring diurnal changes have been planned during the summer of 1961 . It is hoped to monitor simultaneously over 10 to 20 paths of a variety of distances. Some of these paths will lie entirely in the middle latitudes while others will be entirely tropical and others will cross the Arctic icecap.

\section{Effects of Ionospheric Disturbances}

VLF propagation can be affected by a magnetic storm or by a "sudden ionospheric disturbance," (SID). The effect of the magnetic storm is to give an increase in the fluctuations of phase during the night but no serious decrease in amplitude. Fluctuations of up to $5 \mu \mathrm{sec}$ at $16 \mathrm{kc} / \mathrm{s}$ lasting 2 to $3 \mathrm{~min}$ have been reported [Pierce, 1957]. Following the main part of the storm the ionosphere remains disturbed and the diurnal effects are less than normal, recovering over a period (up to a few weeks in some cases). A recording of phase against time during a magnetic storm is shown in figure 7 . This was over the path Rugby-Malta $(2,210 \mathrm{~km})$ for a frequency of $16 \mathrm{kc} / \mathrm{s}$. It will be noted that the steady day phase

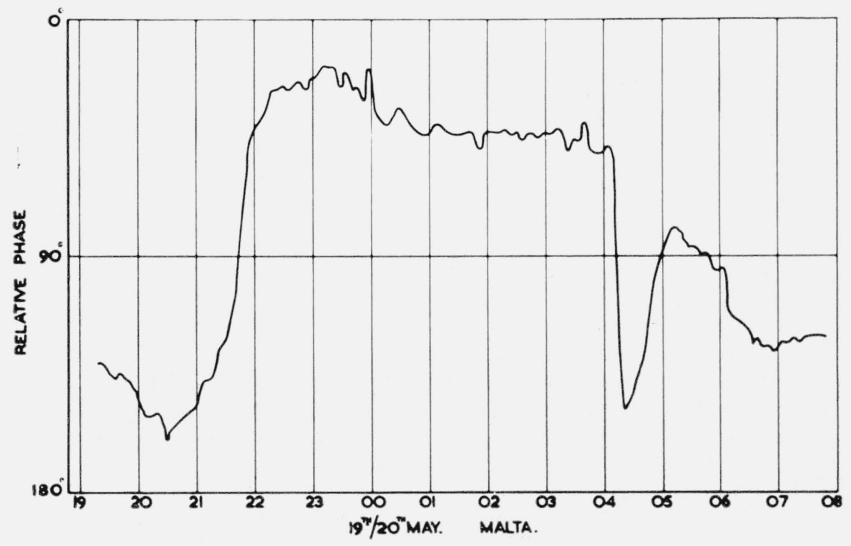

Figure 6. Diurnal variation in phase measured at Malta. is little different from that at night, but that the phase swings have the amplitude expected for a normal day. The features of this record are not yet explained although it is expected that the presence of the overswings at a time when the diurnal changes were small will be significant in the explanation of the overswings on normal days. It should be noted that although the effect of the magnetic storm in this case was to decrease the diurnal effect, there are circumstances in which the effect may be the reverse.

During the solar event of November 1960 the diurnal changes at Malta showed even less change from steady night to steady day, but the overswings were of full amplitude. A simultaneous phase record of GBR taken at Nairobi showed a trapezium of full amplitude. This diurnal change recorded at Nairobi is shown plotted as point 8 of figure 6 . It will be noticed that it lies well on the curve, and is unaffected by the magnetic disturbance.

In order to assess the effects of magnetic storms on the accuracy of a VLF navigational aid, it will be necessary to obtain a wider range of test data by simultaneous and continuous recording on a worldwide basis. This study must determine how widespread and geographically uniform are the effects of a storm, and in particular, how the curve of figure 6 is altered. It may be that the effects of magnetic storms will best be countered by the use of an extra received signal which would help to establish and allow for changes in scale.

Compared with a magnetic storm an SID is of much less consequence. It is associated with a solar flare and effects only the sunlit hemisphere. As far as VLF propagation is concerned it alters the phase change on reflection at the ionosphere so as to give an advance in phase. The effect reaches its maximum in a few minutes and then decreases more slowly to regain a normal level within an hour. In general the amplitude of these changes for a given path is much less than the corresponding diurnal variations, but further data are required, and these could be expected as a byproduct of any monitoring such as that suggested for the study of magnetic effects.

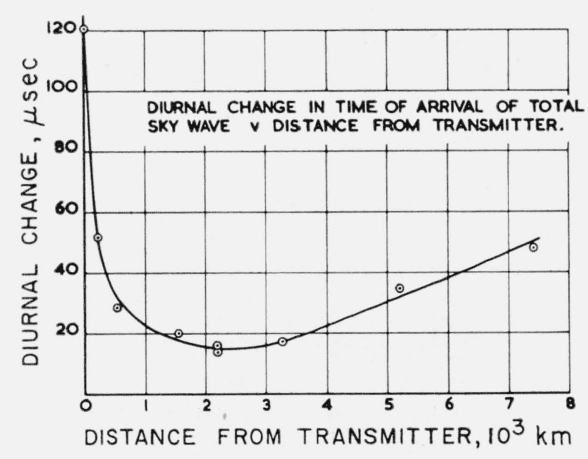

Figure 7. Diurnal change in time of propagation versus distance. 


\section{Airborne Measurements}

In order to assess the potentialities of a VLF navigational aid a series of flights have been made by the Royal Aircraft Establishment, Farnborough. For this work a high stability oscillator was fitted in a Comet aircraft together with receivers which could compare the phase of this oscillator with the signals received from the transmitters GBR (Rugby, $16.0 \mathrm{kc} / \mathrm{s}$ ) and GBZ (Criggion, $15.2 \mathrm{kc} / \mathrm{s}$ ).

Suppose that the aircraft is directly above one of the transmitters and the phase of oscillator and transmitter compared, then if the aircraft moves away the phase of the transmitter will recede because of the time taken for the signal to pass to the aircraft. The recession of transmitter phase is a measure of great circle distance from the transmitter. If the aircraft is on a straight course at a steady speed, a plot of phase change against time will be a smooth curve. In figure 8 is shown a plot of such phase changes measured for GBR and GBZ. The slight scatter of the measured points about the smooth curve is equivalent to an uncertainty in great circle range of 1 nautical mile. Using these two transmitters this consistency has been obtained at distances up to 1,200 nautical miles from the transmitter.

Further airborne experiments have been carried out comparing the phases of the signals from GBR and NBA (Balboa, $18.0 \mathrm{kc} / \mathrm{s}$ ). This has shown that it is possible to track the changes in position of the aircraft using changes in ranges from the two stations. Much of the flying has been over the Mediterranean, and this has shown that the fixes obtained at ranges between 5,000 and 6,000 miles from Balboa on any given flight are internally consistent to within a nautical mile. Their absolute accuracy is not easy to determine because of the difficulty of locating the position of a high flying aircraft. However, a further series of flights is planned during which time theodolite and other photographic methods of determining position will be used.

\section{Conclusion}

The general picture of ionospheric effects on VLF propagation is that there are no fundamental reasons why worldwide navigational aids could not be devised in this frequency band. However, there is a lack of data to fill in the detail of the picture, and only on the basis of such details could one assess the ease and convenience of navigation using such a system. In this field, theory has outstripped experiment, and the need is for an extensive and worldwide monitoring of the phase stability of VLF transmissions over long paths during both normal and disturbed ionospheric conditions.

\section{References}

Budden, K. G., The waveguide mode theory of the propagation of very-low-frequency radio waves, Proc. IRE 45, No. 6, 772-774 (June 1957).

Pierce, J. A., Intercontinental frequency comparison by very-low-frequency radio transmission, Proc. IRE 45, No. 6, 794-806 (June 1957).

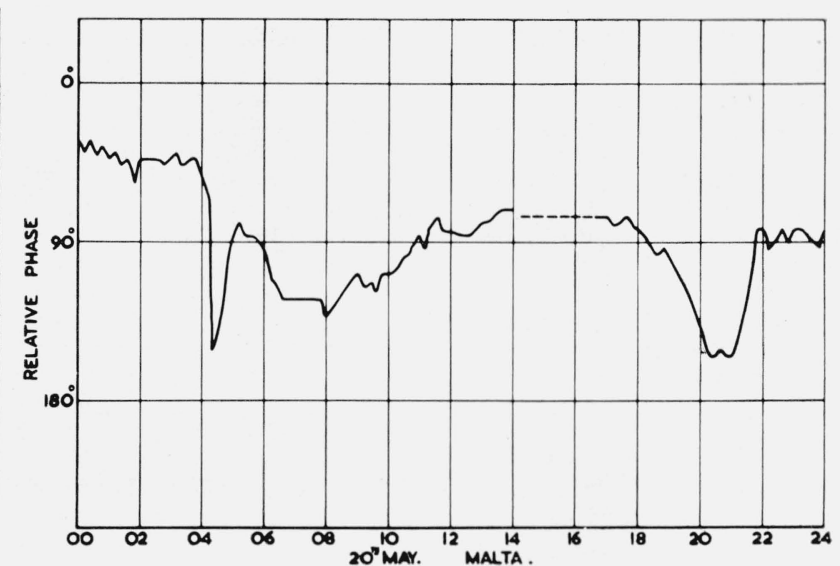

Figure 8. Diurnal variation of relative phase during a magnetic storm.

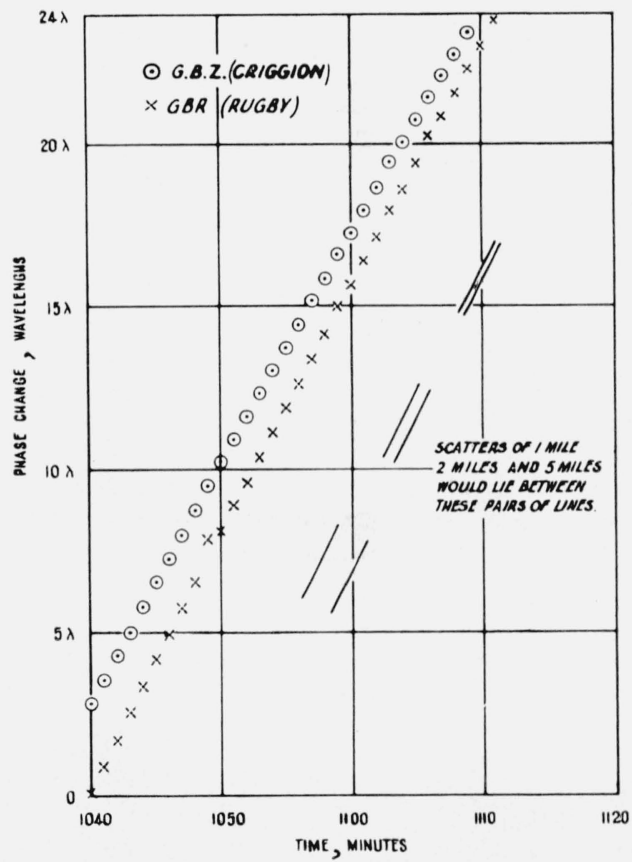

Figure 9. Variation in measured phase against time for a flight on a straight course.

Wait, J. R., The mode theory of VLF ionospheric propagation for finite ground conductivity, Proc. IRE 45, No. 6 , 760-767 (June 1957a).

Wait, J. R., The attenuation vs. frequency characteristics of VLF radio waves, Proc. IRE 45, No. 6, 768-771 (June $1957 \mathrm{~b})$.

Wait, J. R., and A. Murphy, The geometrical optics of VLF sky wave propagation, Proc. IRE 45, No. 6, 754-760 (June 1957). 


\section{Ādditional References}

Bain, W. C., R. N. Bracewell, T. W. Straker, and C. H. Westcott, The ionospheric propagation of radio waves of frequency $16 \mathrm{kc} / \mathrm{s}$ over distances of about $540 \mathrm{~km}$, Proc. Inst. Elec. Engrs. 99, pt IV, No. 3, 250-459 (July 1952).

Bracewell, R. N., The ionospheric propagation of radio waves of frequency $16 \mathrm{kc} / \mathrm{s}$ over distances of about $200 \mathrm{~km}$, Proc. Inst. Elec. Engrs. 99, pt IV, No. 3, 217-228 (July 1952).

Bracewell, R., K. G. Budden, J. A. Ratcliffe, T. W. Straker and $\mathrm{K}$. Weekes, The ionospheric propagation of low- and very-low-frequency radio waves over distances less than $1,000 \mathrm{~km}$, Proc. IRE 98, pt III, 221 (1951). This is an integrating paper surveying the work of the teams working in conjunction with the Cavendish Laboratory at Cambridge.

Wait, J. R., A survey and bibliography of recent research in the propagation of VLF radio waves, NBS Tech. Note 58 (May 1960).

Waynick, A. H., The present state of knowledge of the lower ionosphere, Proc. IRE 45, No. 6, 741-749 (June 1957).

Yabroff, I. W., Reflection at a sharply bounded ionosphere, Proc. IRE 45, No. 6, 750-753 (June 1957).

(Paper 65D6-161) 